\title{
Desempenho e comportamento ingestivo de novilhos holandeses terminados em confinamento com probiótico
}

\author{
Performance and ingestive behavior of confined \\ holstein bull calves fed with probiotic
}

Mikael Neumann ${ }^{[a]}$, Robson Kyoshi Ueno ${ }^{[b]}$, Guilherme Fernando Mattos Leão ${ }^{[c]}$, Esther Devantier Mendes ${ }^{[d]}$, Rodolfo Carletto ${ }^{[e]}$, Itacir Eloi Sandini[f]

[a] Engenheiro agrônomo, Doutor em Zootecnia, Professor adjunto da Universidade Estadual do Centro-Oeste (UNICENTRO), Guarapuava, PR - Brasil, e-mail: mikaelneumann@hotmail.com

[b] Médico veterinário, Mestre em Agronomia, Doutorando do Programa de Pós-Graduação em Zootecnia da Universidade Federal do Rio Grande do Sul (UFRGS), Porto Alegre, RS - Brasil, e-mail: robsonueno@hotmail.com

[c] Acadêmico do curso de Medicina Veterinária, Universidade Estadual do Centro-Oeste (UNICENTRO), Guarapuava, PR Brasil, e-mail: gfleao@hotmail.com

[d] Médica veterinária, Mestranda do Programa de Pós-Graduação em Agronomia, Universidade Estadual do Centro-Oeste (UNICENTRO), Guarapuava, PR - Brasil, e-mail: esthermendesmedvet@hotmail.com

[e] Engenheiro agrônomo, Mestrando do Programa de Pós-Graduação em Agronomia, Universidade Estadual do CentroOeste (UNICENTRO), Guarapuava, PR - Brasil, e-mail: rodolfocarletto@hotmail.com

[f] Engenheiro agrônomo, Doutor em Agronomia, Professor adjunto da Universidade Estadual do Centro-Oeste (UNICENTRO), Guarapuava, PR - Brasil, e-mail: isandini@hotmail.com

\section{Resumo}

A indústria de nutrição animal constantemente insere no mercado produtos que visam melhorar o desempenho animal. Dentre eles, as cepas de leveduras têm grande representatividade, tornando-se necessária a verificação de sua eficácia. Objetivou-se avaliar o desempenho alimentar, comportamento ingestivo e digestibilidade aparente da matéria seca de novilhos da raça holandesa, em fase de terminação em confinamento com probiótico. 0 delineamento experimental foi inteiramente casualizado com seis repetições, utilizando 24 novilhos, com a inclusão ou não do probiótico contendo leveduras secas vivas Saccharomyces cerevisiae cepa $\mathrm{KA}_{500}\left(2 \times 10^{10} \mathrm{UFC} . \mathrm{g}^{-1}\right)$. Não houve diferença significativa $(P>0,05)$ para nenhuma das variáveis testadas. 0 ganho de peso médio diário atingiu valor médio de $1,285 \mathrm{~kg}^{-1}$.animal ${ }^{-1}$. $\mathrm{dia}^{-1}$, enquanto que o consumo de matéria seca apresentou valor médio de $7,54 \mathrm{~kg}^{-1}$.animal-1.dia-1. 0 valor médio da conversão alimentar foi de 6,06 kg de matéria seca por kg de peso ganho, e o consumo médio por cada $100 \mathrm{~kg}$ de peso vivo foi de 2,32. Para as variáveis relacionadas ao comportamento ingestivo, como tempo de ruminação, tempo em ócio, tempo de alimentação e tempo de ingestão de água, obtiveram-se tempos médios de 6,80; 13,$97 ; 3,07$ e 0,17 horas.dia $^{-1}$, respectivamente. 0 uso do probiótico não exerceu diferença significativa para 
o desempenho alimentar, comportamento ingestivo e digestibilidade aparente da dieta de novilhos da raça holandesa em confinamento.

Palavras-chave: Consumo. Digestibilidade. Leveduras. Saccharomyces cerevisiae.

\section{Abstract}

The animal nutrition industry constantly launches products to improve animal performance, including a large number ofyeast strains, which makes it necessary to check their effectiveness. The objective of the present study was to evaluate the feed intake performance behavior and apparent dry matter digestibility in Holstein bull calves fed with probiotics and finished in confinement. The experimental design was completely randomized with 6 replicates using 24 bull calves with the inclusion or not of dried Saccharomyces cerevisiae $K A_{500}\left(2 \times 10^{1}\right.$ $\left.{ }^{0} \mathrm{CFU}{ }^{-1}\right)$ in their diet. There was no significant difference $(P>0.05)$ for any of the variables tested. The average daily weight gain was $1.285 \mathrm{~kg}^{-1}$.animal ${ }^{-1}$.day ${ }^{-1}$, while the dry matter intake averaged $7.54 \mathrm{~kg}^{-1}$. animal ${ }^{-1}$.day ${ }^{-1}$. The average feed conversion was $6.06 \mathrm{~kg}$ of dry matter per $\mathrm{kg}$ weight gain, and the average consumption per $100 \mathrm{~kg}$ of live weight was $2.32 \mathrm{~kg}$. Rumination, leisure, feeding and drinking times averaged 6.80, 13.97, 3.07 and 0.17 hours.day ${ }^{-1}$, respectively. The use of probiotic did not cause significant difference to the feed intake performance behavior and apparent digestibility of confined Holstein calves.

Keywords: Intake. Digestibility. Yeast. Saccharomyces cerevisiae.

\section{Introdução}

Na bovinocultura de leite do Brasil, o destino dos bezerros ainda é um grande entrave. Os bezerros de raças leiteiras, em sua maioria, ainda não são utilizados para o corte, sendo geralmente sacrificados logo após o nascimento. Caso contrário, estão fadados a um futuro incerto, submetidos a condições de subnutrição e insalubridade. Essas situações infringem as premissas de ética e bem estar na criação de animais.

O desprezo a esses animais incorre no desperdício de uma alternativa que poderia gerar uma oferta maior de carne no mercado e, por conseguinte, ser fonte de renda extra do produtor ou levar ao surgimento de outro segmento no sistema de produção de carne nacional. (TEIXEIRA et al., 2000; JORGE et al., 2009).

Entretanto, como pertencem a rebanhos para produção de leite, devem ser desmamados muito precocemente, necessitando de cuidados e tecnologias diferenciadas desde o nascimento para tornarem-se viáveis bioeconomicamente (FREITAS NETO, 2009). Na literatura, são relatados bons resultados no desempenho e na carcaça desses animais (TEIXEIRA et al., 2000; MAGALHÃES et al., 2005).

Uma das principais alternativas pesquisadas e utilizadas para otimizar o desempenho em ruminantes são os aditivos probióticos, constituídos por preparos de culturas de microorganismos vivos e/ ou seus metabólitos que, em teoria, são benéficos por prevenir a colonização de microorganismos indesejáveis ou promover o reestabelecimento da microbiota normal do trato gastrointestinal, não deixando resíduos tóxicos nas carcaças (OYETAYO et al., 2005; BERCHIELLI et al., 2011).

Inúmeros são os microorganismos utilizados como probióticos, sempre com o intuito de produzir efeitos benéficos ao hospedeiro. Em bovinos, pode se observar o efeito promotor de crescimento e engorda, melhoria na eficiência alimentar, imunoestimulante e minimizador de estresse. Estes efeitos são promovidos por diversos mecanismos de ação conhecidos e outros em fase de estudo (FREITAS NETO, 2009).

O principal objetivo da suplementação com probiótico é a manipulação da fermentação ruminal, visando um incremento produtivo (FERELI et al., 2010). A suplementação com bactérias utilizadoras 
e/ou produtoras de lactato em dietas de bovinos têm melhorado o desempenho dos animais (GALYEAN et al., 1989).

As leveduras vivas (Saccharomyces cerevisiae) possuem diversas funções, dentre as quais se pode citar: o auxílio na manutenção do pH no rúmen via estímulo de bactérias utilizadoras de lactato; aumento da maturidade do rúmen por favorecer o estabelecimento microbiano, e aumento da degradação da fibra pelo favorecimento das bactérias fibrolíticas (ROSE, 1997; BERCHIELLI et al., 2011).

Os efeitos benéficos com a utilização de leveduras se dão pela sua capacidade de estimular a multiplicação de bactérias interessantes ao rúmen, como as bactérias anaeróbicas totais, celulolíticas e utilizadoras de ácido láctico. Estes eventos contribuem para a melhora dos processos de digestão da fibra, com consequente efeito positivo sobre a ingestão de matéria seca, ganho de peso, conversão alimentar e menor incidência de distúrbios metabólicos (DOREAU; JOUANY, 1998; TRICARICO, 2005).

Entretanto, na literatura, os resultados envolvendo o uso de leveduras como aditivos probióticos são bastante variáveis. A inconsistência de resultados pode ocorrer em função da variação entre concentração e combinação dos microorganismos, categoria animal, tipos de dieta e formas de fornecimento, fazendo-se necessário o estudo do efeito de probióticos frente a diferentes dietas para animais em alto desafio, como na fase de recria/terminação de novilhos de raças leiteiras em estabulamento total.

Diante disso, objetivou-se com o presente trabalho avaliar o desempenho animal, comportamento ingestivo e digestibilidade aparente da dieta fornecida a novilhos da raça holandesa, em fase de terminação em confinamento com ou sem o fornecimento de probiótico.

\section{Material e métodos}

$\mathrm{O}$ experimento foi conduzido nas instalações do Núcleo de Produção Animal (NUPRAN) do Setor de Ciências Agrárias e Ambientais da Universidade Estadual do Centro-Oeste (UNICENTRO), em Guarapuava-PR, e em parceria com o Instituto Paranaense de Assistência Técnica e Extensão Rural (EMATER-PR), Cooperativa Agrária Agroindustrial e Kera Nutrição Animal.
As variáveis mensuradas para avaliar o efeito do fornecimento do probiótico contendo leveduras vivas para os novilhos foram: ganho de peso médio diário (GMD), consumo de matéria seca diário (CMSD), conversão alimentar (CA), comportamento ingestivo (CI) e digestibilidade aparente da matéria seca (DMS) da dieta.

0 delineamento experimental foi inteiramente casualizado, constituindo-se de dois tratamentos (com ou sem probiótico) e seis repetições. Cada repetição foi representada por uma baia com dois animais. 0 probiótico foi fornecido na dose de 10 g.animal ${ }^{-1} \cdot$ dia $^{-1}$, composto por Saccharomyces cerevisiae cepa $\mathrm{KA}_{500}\left(2 \times 10^{10} \mathrm{UFC}^{-1} \mathrm{~g}^{-1}\right)$.

Foram utilizados 24 novilhos da raça Holandesa com idade média inicial de 192 dias de vida e peso vivo médio inicial de $221 \mathrm{~kg} \pm 10 \mathrm{~kg}$. Os animais foram alojados em recintos de confinamento, distribuídos aleatoriamente em 12 baias semicobertas com área de $15 \mathrm{~m}^{2}$ cada $(2,5 \mathrm{~m} \mathrm{x} \mathrm{6,0} \mathrm{m),} \mathrm{comedou-}$ ro de concreto e bebedouro metálico regulado por boia automática.

0 experimento teve duração de 210 dias, sendo os 35 dias iniciais um período de adaptação dos animais às dietas e instalações experimentais e, sequencialmente, cinco períodos de 35 dias cada. Os animais foram pesados, após jejum de sólidos durante 12 horas, no início e fim do período experimental, com pesagens intermediárias a cada 35 dias.

As dietas experimentais foram reguladas diariamente para proporcionar consumo de $1,5 \%$ do peso vivo em alimento concentrado, sendo o restante do consumo composto por alimento volumoso na proporção de $50 \%$ de silagem de milho e $50 \%$ de feno de aveia, com fornecimento ad libitum. Na preparação do concentrado foram utilizados os seguintes alimentos: farelo de soja, casca de soja, farelo de trigo, radícula de malte, cevada, grãos de milho moídos, gérmen de milho, calcário calcítico, fosfato bicálcico, uréia pecuária, premix vitamínico e mineral, sal comum e monensina sódica.

Foram realizadas amostras compostas da silagem de milho, do feno de aveia e do concentrado de cada tratamento durante os períodos de avaliação do confinamento. As amostras dos alimentos foram levadas à estufa de ar forçado a $55{ }^{\circ} \mathrm{C}$ até atingir peso constante, para determinação da matéria parcialmente seca. As amostras pré-secas foram 
moídas em moinho tipo Wiley com peneira de $1 \mathrm{~mm}$ de diâmetro e conduzidas, posteriormente, para análise bromatológica.

Nas amostras pré-secas, foram determinados os teores de matéria seca total em estufa de secagem e esterilização a $105^{\circ} \mathrm{C}$, de proteína bruta (PB) pelo método micro Kjedahl, de matéria mineral (MM) por incineração a $550{ }^{\circ} \mathrm{C}$ e de extrato etéreo (EE) conforme técnicas descritas em AOAC (1995). Os teores de fibra em detergente neutro (FDN) foram obtidos conforme método de Robertson, Lewis e Van Soest (1991) com a enzima $\alpha$-amilase termoestável e de fibra em detergente ácido (FDA), segundo Goering e Van Soest (1970). Os nutrientes minerais foram analisados conforme metodologia descrita por Tedesco et al. (1995).

Na Tabela 1 são apresentados os teores de nutrientes médios da ração, calculados conforme a análise dos alimentos e percentual de inclusão na ração.

Tabela 1 - Teor de nutrientes na ração, na média do período experimental

\begin{tabular}{cc}
\hline Parâmetro & Teor (\%) \\
\hline Matéria seca & 68,99 \\
Proteína bruta & 15,37 \\
Extrato etéreo & 3,52 \\
Fibra em detergente neutro & 38,53 \\
Fibra em detergente ácido & 20,77 \\
Matéria mineral & 6,81 \\
Cálcio & 0,86 \\
Fósforo & 0,40 \\
Potássio & 0,91 \\
Magnésio & 0,28 \\
\hline
\end{tabular}

Fonte: Dados da pesquisa.

Os animais foram alimentados duas vezes ao dia, às 6:00 e às 17:30 horas, onde o consumo voluntário dos alimentos foi registrado diariamente, pesando-se a quantidade oferecida e as sobras do dia anterior. 0 ajuste no fornecimento da quantidade de silagem de milho e feno de aveia foi realizado diariamente, considerando uma sobra de $5 \%$ da matéria seca oferecida em relação à consumida, ao passo que a quantidade de concentrado oferecida foi regulada para representar $1,5 \%$ do peso vivo médio dos animais em cada período.
A avaliação do comportamento ingestivo dos animais foi realizada em um período intermediário do confinamento (meados do terceiro período), durante 72 horas contínuas de observação. As mensurações foram realizadas por 12 observadores por turno, revezando-se a cada 6 horas. As leituras foram tomadas em intervalos regulares de 3 minutos. As variáveis analisadas resultaram da mensuração do tempo despendido para as atividades de ócio, ruminação, ingestão de água e alimentação, expressas em horas por dia.

Durante a avaliação do comportamento dos animais, procedeu-se a avaliação da DMS das dietas experimentais. Para tanto, coletou-se a produção total de fezes de cada unidade experimental no momento da eliminação da excreta, as quais foram pesadas e amostradas em cada turno de 6 horas e, posteriormente, armazenadas sob refrigeração em sacos plásticos individuais. Ao fim do período de 72 horas, as fezes de cada unidade experimental foram homogeneizadas para a formação de uma amostra composta representativa dos 3 dias de avaliação. As amostras foram secadas em estufas de ar forçado a $55^{\circ} \mathrm{C}$ até obterem peso constante.

Nas amostras das fezes secas, foram determinados os coeficientes de digestibilidade aparente (CD), segundo Prates (2007), através da expressão $\mathrm{CD}(\%)=(\mathrm{g}$ de MS consumida - $\mathrm{g}$ de MS excretada $)$ / g de MS consumida X 100.

Os dados relativos ao desempenho animal, $\mathrm{CI}$ e DMS, foram submetidos a análise de variância com probabilidade de erro a $5 \%$ pelo teste F, por intermédio do programa estatístico SAS (1993). Os dados de desempenho também foram submetidos à análise de regressão polinomial, considerando a variável período (175 dias), por intermédio do procedimento proc reg do programa SAS (1993).

\section{Resultados e discussão}

A adição da levedura em dieta de ruminantes possui, teoricamente, diversas vantagens. Cabe ressaltar que, a nível ruminal, provoca, em virtude de seu consumo de oxigênio, uma maior degradação da fibra, causada pelo aumento expressivo de bactérias anaeróbicas, especialmente as celulolíticas (DESNOYERS et al., 2009; PIRES, 2011). 
Além disso, a menor variação de $\mathrm{pH}$ garante maior estabilidade no ambiente ruminal, que se deve ao consumo do ácido láctico do meio pelas leveduras (WILLIAMS et al., 1991; WALLACE et al., 1994; PIRES, 2011). Quanto aos aspectos de desempenho, no entanto, a inclusão de levedura ainda apresenta resultados variáveis na literatura, embora seja foco de pesquisa há décadas. A maioria dos trabalhos apresentam resultados não significativos (QUEIRÓZ et al., 2004; GATTASS et al., 2008; HOLTSHAUSEN et al., 2010).

No presente estudo, conforme a Tabela 2, não foram encontradas interações significativas entre tratamentos e períodos de avaliação para nenhuma das variáveis mensuradas. Não houve diferença considerável entre os tratamentos para o GMD $(\mathrm{P}=0,1720)$, apresentando valores médios de 1,243 para o tratamento COM levedura e $1,327 \mathrm{~kg}^{-1}$.ani$\mathrm{mal}^{-1}$.dia ${ }^{-1}$ para o tratamento SEM levedura. Na CA tampouco houve diferença significativa entre tratamentos $(P=0,4476)$, sendo os valores médios de 6,18 contra 5,94 kg de MS por kg de peso vivo referentes a COM e SEM levedura, respectivamente. Também não foram observadas diferenças entre os tratamentos para o CMSD e para o CMSPV, sendo que os valores médios encontrados foram de $7,54 \mathrm{~kg}^{-1}$ ani$\mathrm{mal}^{-1}$. $\mathrm{dia}^{-1}$ e $2,32 \%$ do peso vivo, respectivamente.

Em contrapartida, estudos recentes vêm demonstrando os efeitos benéficos da levedura. Ramsing et al. (2009) constataram que vacas recebendo leveduras, tanto primíparas quanto multíparas, no período de transição, melhoraram a ingestão de matéria seca no período pré-parto e o desempenho pós-parto.

Tabela 2 - Efeito do uso de probiótico (Saccharomyces cerevisiae) sobre o desempenho de novilhos holandeses terminados em confinamento, conforme período de avaliação

\begin{tabular}{|c|c|c|c|c|c|c|}
\hline \multirow[b]{2}{*}{ Probiótico } & \multicolumn{5}{|c|}{ Período de confinamento (dias) } & \multirow[b]{2}{*}{ MÉDIA } \\
\hline & $\begin{array}{c}1^{\circ} \\
(1-35) \\
\end{array}$ & $\begin{array}{c}2^{\circ} \\
(36-70) \\
\end{array}$ & $\begin{array}{c}3^{\circ} \\
(71-105) \\
\end{array}$ & $\begin{array}{c}4^{\circ} \\
(105-140) \\
\end{array}$ & $\begin{array}{c}5^{\circ} \\
(141-175) \\
\end{array}$ & \\
\hline \multicolumn{7}{|c|}{ Ganho de peso médio diário (kg-1.animal-1.dia-1) } \\
\hline Com & 1,200 & 1,269 & 1,200 & 1,164 & 1,382 & $1,243 \mathrm{a}$ \\
\hline Sem & 1,368 & 1,411 & 1,355 & 1,182 & 1,318 & $1,327 \mathrm{a}$ \\
\hline Média & 1,284 & 1,340 & 1,278 & 1,173 & 1,350 & \\
\hline \multicolumn{7}{|c|}{ *Equação regressão GMD= 1,285 kg dia-1 (R2: 0,0012; CV: 12,6\%; P<0,7899) } \\
\hline \multicolumn{7}{|c|}{ Conversão alimentar (kg de MS por kg de peso vivo) } \\
\hline Com & 5,25 & 5,33 & 6,56 & 7,20 & 6,54 & $6,18 \mathrm{a}$ \\
\hline Sem & 4,40 & 4,91 & 6,10 & 7,40 & 6,89 & $5,94 \mathrm{a}$ \\
\hline Média & 4,83 & 5,12 & 6,33 & 7,30 & 6,72 & \\
\hline \multicolumn{7}{|c|}{ *Equação regressão $C A=4,2299+0,0173 D(R 2: 0,3217 ; C V: 20,8 \% ; P<0,0001)$} \\
\hline \multicolumn{7}{|c|}{ Consumo de matéria seca diário (kg-1.animal-1.dia-1 } \\
\hline Com & 5,89 & 6,45 & 7,75 & 8,28 & 8,84 & 7,44 a \\
\hline Sem & 5,85 & 6,85 & 8,11 & 8,49 & 8,90 & $7,64 \mathrm{a}$ \\
\hline Média & 5,87 & 6,65 & 7,93 & 8,39 & 8,87 & \\
\hline \multicolumn{7}{|c|}{ *Equação regressão CMSD= 5,2420 + 0,0218D (R2: 0,6187; CV: 11,4\%; P<0,0001) } \\
\hline \multicolumn{7}{|c|}{ Consumo por $100 \mathrm{~kg}$ de peso vivo } \\
\hline Com & 2,48 & 2,31 & 2,40 & 2,27 & 2,20 & $2,33 \mathrm{a}$ \\
\hline Sem & 2,44 & 2,38 & 2,42 & 2,24 & 2,14 & $2,32 \mathrm{a}$ \\
\hline Média & 2,46 & 2,35 & 2,41 & 2,26 & 2,17 & \\
\hline \multicolumn{7}{|c|}{ *Equação regressão CMSPV= 2,5255 - 0,0019D (R2: 0,1001; CV: 12,3\%; P<0,0138) } \\
\hline
\end{tabular}

Legenda: * D: Período de confinamento variando de 1 a 175 dias.

Nota: Médias, seguidas por letras minúsculas diferentes, na coluna, diferem entre si pelo Test $\mathrm{F}$ a $5 \%$.

Fonte: Dados da pesquisa. 
Neumann et al. (2013), por outro lado, atestaram que o tratamento contendo leveduras vivas foi superior no ganho de peso $\left(1,235 \mathrm{vs.} 1,099 \mathrm{~kg}^{-1}\right.$.animal $^{-1} \cdot$ dia $\left.^{-1}\right)$ e na conversão alimentar $(7,22$ vs. 7,95 kg de matéria seca ingerida para kg de ganho de peso) de bovinos de corte confinados.

Na Tabela 3 encontram-se os valores relativos às variáveis comportamentais de novilhos terminados em confinamento COM ou SEM probiótico. Não se obteve diferença significativa para o tempo de ruminação, tempo em ócio, tempo de alimentação e tempo de ingestão de água entre os tratamentos COM ou SEM probiótico, dispensando ocupação de 6,80; 13,$97 ; 3,07$ e 0,17 horas. dia $^{-1}$ para as respectivas variáveis. A DMS também foi similar entre os animais alimentados ou não com o suplemento probiótico, apresentando valor médio de 67,84\%.

Tabela 3 - Efeito do uso de probiótico (Saccharomyces cerevisiae) sobre o comportamento ingestivo e digestibilidade aparente da dieta de novilhos holandeses terminados em confinamento

\begin{tabular}{lccc}
\hline \multirow{2}{*}{ Variáveis Comportamentais } & \multicolumn{3}{c}{ Probiótico } \\
\cline { 2 - 5 } & Com & Sem & $\begin{array}{c}\text { Valores } \\
\text { de P }\end{array}$ \\
\hline Tempo de ruminação, horas.dia-1 & 6,90 & 6,70 & 0,8798 \\
Tempo de ócio, horas.dia-1 & 13,75 & 14,19 & 0,8106 \\
Tempo de alimentação, horas.dia-1 & 3,20 & 2,95 & 0,6502 \\
Tempo de ingestão de água, horas.dia-1 & 0,16 & 0,18 & 0,3376 \\
Digestibilidade aparente, \% da MS & 68,07 & 67,61 & \\
\hline
\end{tabular}

Nota: *Não houve diferença significativa entre os tratamentos $(P<0.05)$. Fonte: Dados da pesquisa.

Outros testes do efeito da inclusão de levedura no comportamento ingestivo também não obtiveram diferença significativa para os tempos de ruminação, ócio, alimentação e ingestão de água (SEGABINAZZI et al., 2011; NEUMANN et al., 2013). Porém, Neumann et al. (2013) observaram um aumento da frequência de alimentações diárias de animais que consumiam leveduras vivas, sugerindo como possível resposta para este reflexo o aumento na taxa de passagem da dieta pelo trato gastrointestinal.

Zeoula et al. (2008) testaram a inclusão de ionóforos e probióticos em diferentes dietas para bovinos da raça Holandesa e búfalos da raça Murrah, encontrando resultados de coeficiente de digestibilidade aparente $(63,3 \%)$, para bovinos alimentados com levedura, próximos aos encontrados na presente pesquisa $(67,61 \%)$. Também, não se evidenciou diferenças significativas para a DMS.

Goes et al. (2005), em revisão de literatura, consideram as leveduras aditivos potencialmente utilizados, e justificam a inconstância de resultados na inclusão desses probióticos na nutrição de ruminantes pela falta de conhecimentos acerca de mecanismos fisiológicos específicos e dos requerimentos destes microrganismos. É necessário, portanto, que se realizem mais estudos elucidando tais pontos.

\section{Conclusão}

O uso do probiótico à base de leveduras vivas (Saccharomyces cerevisiae) na dieta de novilhos Holandeses em fase de terminação em confinamento não apresentou efeito significativo sobre o desempenho animal, comportamento ingestivo e digestibilidade aparente da matéria seca.

\section{Referências}

ASSOCIATION OF OFFICIAL ANALYTICAL CHEMISTS AOAC. Official methods of analysis. 16. ed. Washington, DC: AOAC, 1995.

BERCHIELLI, T. T. et al. Nutrição de ruminantes. 2. ed. Jaboticabal: FUNEP, 2011.

DESNOYERS, M. et al. Meta-analysis of the influence of Sacharomyces cerevisiae supplementation on ruminal parameters and milk production of ruminants. Journal of Dairy Science, v. 92, n. 4, p. 1620-1632, 2009. doi:10.3168/jds.2008-1414.

DOREAU, M.; JOUANY, J. P. Effect of a Saccharomyces cerevisae culture on nutrient digestion in lactating dairy cows. Journal of Dairy Science, v. 81, n. 12, p. 32143221, 1998. PMid:9891266.

FERELI, F. et al. Monensina sódica e Saccharomyces cerevisiae em dietas para bovinos: fermentação ruminal, digestibilidade dos nutrientes e eficiência de síntese microbiana.

Revista Brasileira de Zootecnia, v. 39, n. 1, p. 183-190, 2010. doi:10.1590/S1516-35982010000100024. 
FREITAS NETO, M. D. Desempenho de bovinos mestiços leiteiros alimentados com diferentes níveis de concentrado na recria e terminação. 2009. 37 f. Dissertação (Mestrado em Ciência Animal) - Universidade Federal de Goiás, Goiânia, 2009.

GALYEAN, M. L.; HUBBERT, M. E. Rationale for use and selection of ionophores in ruminant nutrition. In: SOUTHWEST NUTRITION AND MANAGEMENT CONFERENCE, 1989, Tucson. Proceedings... Tucson: University of Arizona, 1989. p. 64-81.

GATTASS, C. B. A. et al. Consumo, digestibilidade aparente e ganho de peso em Bovinos de corte confinados e suplementados com cultura de levedura (Saccharomyces cerevisiae cepa 1026). Ciência Animal Brasileira, v. 9, n. 3, p. 535-542, 2008.

GOERING, H. K.; VAN SOEST, P. J. Forage fiber analysis (apparatus reagents, procedures, and some applications). Agricultural Handbook no. 379. Washington, DC: U.S. Agricultural Research Service, 1970.

GOES, R. H. T. B. et al. Utilização de aditivos alimentares microbianos na alimentação de bovinos de corte e leite: revisão. Arquivos de Ciências Veterinárias e Zoologia da UNIPAR, v. 8, n. 1, p. 47-56, 2005.

HOLTSHAUSEN, L.; BEAUCHEMIN, K. A. Supplementing barley-based dairy cow diets with Saccharomyces cerevisiae. The Professional Animal Scientist, v. 26, n. 3, p. 285-289, 2010.

JORGE, J. R. V. et al. Gordura protegida sobre o desempenho, carcaça e composição química da carne de novilhos holandês. Archivos de Zootecnia, v. 58, n. 223, p. 371382, 2009. doi:10.4321/S0004-05922009000300006.

MAGALHÃES, K. A. et al. Desempenho, composição física e características da carcaça de novilhos alimentados com diferentes níveis de casca de algodão, em confinamento. Revista Brasileira de Zootecnia, v. 34, n. 6, p. 24662474, 2005. doi:10.1590/S1516-35982005000700035.

NEUMANN, M. et al. Leveduras vivas (Saccharomyces cerevisiae) sobre o desempenho de novilhos terminados em confinamento e as características da carne e da carcaça. Revista Acadêmica: Ciências Agrárias e Ambientais, v. 11, n. 1, p. 75-85, 2013. doi:10.7213/academica.7758.

OYETAYO, V. O.; OYETAYO, F. L. Potential of probiotics as biotherapeutic agents targeting the innate immune system. African Journal of Biotechnology, v. 4, n. 2, p. 123127, 2005. doi:10.5897/AJB2005.000-3025.
PIRES, L. C. B. Utilização de leveduras na alimentação de ruminantes. Cadernos de Pós-Graduação da Fazu, v. 2, p. 1-8, 2011.

PRATES, E. R. Técnicas de pesquisa em nutrição animal. Porto Alegre: Editora da UFRGS, 2007.

QUEIROZ, R. C. et al. Uso de produto à base de enzima e levedura na dieta de bovinos: digestibilidade dos nutrientes e desempenho em confinamento. Revista Brasileira de Zootecnia, v. 33, n. 6, p. 1548-1556, 2004. doi:10.1590/ S1516-35982004000600022.

RAMSING, E. M. et al. Effects of yeast culture on peripartum intake and milk production of primiparous and multiparous holstein cows. The Professional Animal Scientist, v. 25, n. 4, p. 487-495, 2009.

ROSE, A. H. Yeast, a microorganism for all species: a theoretical look at its mode of action. In: LYONS, T. P. (Ed.). Biotechnology in the feed industry. Nicholasville: Alltech Technical Publications, 1997. p. 113-118.

SAS INSTITUTE. User's guide: statistics. Version 6. 4. ed. North Caroline: SAS/STAT, 1993.

SEGABINAZZI, L. R. et al. Behavior patterns of cows with Charolais or Nellore breed predominance fed diets with plant extract or monensin sodium. Revista Brasileira de Zootecnia, v. 40, n. 12, p. 2954-2962, 2011. doi:10.1590/ S1516-35982011001200044.

TEDESCO, M. J. et al. Análises de solo, plantas e outros materiais. 2. ed. Porto Alegre: Editora da UFRGS, 1995. (Boletim Técnico, n. 5).

TEIXEIRA, J. C. et al. Aproveitamento do macho leiteiro utilizando dietas à base de amireia 45s. II. Desempenho. Ciência e Agrotecnologia, v. 24, n. 1, p. 203-207, 2000.

TRICARICO, J. M. Otimizando a função ruminal: benefícios de Beef-Sacc na produtividade animal. In: SIMPÓSIO BRASILEIRO DA INDÚSTRIA DE ALIMENTAÇÃO ANIMAL, 2., 2005, Curitiba. Anais... Curitiba: Simpósio Brasileiro Alltech, 2005. p. 119-126.

ROBERTSON, J. B.; LEWIS, B. A.; VAN SOEST, P. J. Methods for dietary fiber, neutral detergent fiber, and nonstarch polysaccharides in relation to animal nutrition. Journal of Dairy Science, v.74, n.10, p.3583-3597, 1991. doi:10.3168/jds.S0022-0302(91)78551-2.

WALLACE, R. J. Ruminal microbiology, biotechnology and ruminant nutrition: progress and problems. Journal of Animal Science, v. 72, n. 11, p. 2992-3003, 1994. PMid:7730195. 
WILLIAMS, P. E. et al. Effects of the inclusion of yeast culture (Saccharomyces cereviseae) plus growth medium) in the diet of dairy cows on milk yield and forage degradation and fermentation patterns in the rumen of steers. Journal of Animal Science, v. 69, n. 7, p. 3016-3026, 1991. PMid:1885411.
ZEOULA, L. M. et al. Digestibilidade parcial e total de rações com a inclusão de ionóforo ou probiótico para bubalinos e bovinos. Revista Brasileira de Zootecnia, v. 37, n. 3, p. 563-571, 2008. doi:10.1590/ S1516-35982008000300023.

Recebido: 28/02/2013

Received: 02/28/2013

Aprovado: 20/12/2013

Approved: 12/20/2013 